\title{
Original
}

\section{Biomechanical Evaluation of Anterior Lumbar Interbody Fusion with Threaded Cage}

\author{
Toshiyuki Shirahata ${ }^{1)}$, Yutaka Hiraizumi ${ }^{1}$, Hideyo MiYaOKa ${ }^{1}$, \\ Koichi KobaYASHI ${ }^{2)}$, Hiroyuku TAKeISHI $^{3)}$ and Yoshisada SATO $^{4)}$
}

\begin{abstract}
Anterior lumbar interbody fusion (ALIF), under laparoscope or retroperitoneoscope is a well accepted procedure for treatment of degenerative spinal abnormalities. Interbody fusion cages have been shown to promote good initial fixation in a variety of animal models, however few studies have examined the effects of the type of cage or direction of insertion on initial fixation strength. The present study biomechanically measured the initial fixation strength of ALIF using a threaded interbody fusion cage on fresh porcine spine. We performed two studies; Study 1 compared the effects of the number and the direction of insertion of cylindrical cages at the L4/5,5/6 level on stiffness, and Study 2 investigated the effects of the type of cage and number using two types of cages (cylindrical and conical cages) at the L6/S1 level. In Study 1, there was significantly more stiffness with flexion in the single anterior group, the double anterior group, and the single anterolateral group compared to the control group (no cage insertion). In addition, stiffness was significantly better in the single anterolateral group than in the single anterior group. There was no differentces between any of the groups in any loading mode. In Study 2, for extension, there was significantly better stiffness in the groups that received cylindrical or conical cages compared to no-insertion controls. In lateral bending, the two cylindrical cage groups had significantly better stiffness than controls. There were no differences in flexion and axial torsion between any of the groups. Again, there were no significant differences between any of the groups in any loading mode.
\end{abstract}

Key words : ALIF, threaded fusion cage, primary stiffness, biomechanics

\section{Introduction}

Interbody fusion is frequently used to treat degenerative diseases of intervertebral discs of lumbar vertebrae. The conventional approach to interbody fusion is to use bone from the patient, taken from the ilium, with fixation of intervertebral disks using spacers consisting of artificial bone. However, with interbody fusion solely with transplanted bone, a decrease

\footnotetext{
1) Department of Orthopedic Surgery, Showa University School of Medicine, 1-5-8, Hatanodai, Shinagawa-ku, Tokyo 142-8666, Japan.

2) Department of Health Sciences, Niigata University School of Medicine.

${ }^{3)}$ Faculty of Engineering, Chiba Institute of Technology.

${ }^{4)}$ Mon-naka Orthopedic Clinic.
} 
in intervertebral height due to dislodging and collapse of transplanted bone has led to problems with fixation and incomplete bone union ${ }^{1)}$. The threaded interbody fusion cage was developed to provide stronger fixation and early ambulation ${ }^{2)}$. Anterior lumbar interbody fusion (ALIF), under laparoscope or retroperitoneoscope, has more recently become a well accepted procedure for treatment of degenerative spinal abnormalities. Good initial fixation using an interbody fusion cage has been reported with the use of ALIF $^{3-5}$, posterior lumbar interbody fusion $(\text { PLIF })^{6,7)}$, and when fixation is added posteriorly with a pedicle screw system ${ }^{8-10)}$. However, few studies have examined the effects of the type of interbody fusion cage or direction of insertion on initial fixation strength when used as a stand-alone device ${ }^{11)}$. The present study measured the initial fixation strength of ALIF using a threaded interbody fusion cage on fresh porcine spine. We evaluated insertion of a cylindrical threaded cage to the porcine L4/5,5/6 level (equivalent to human L3/4,4/5 level) and of either a cylindrical or a conical threaded cage to porcine L6/S1 level (human L5/S1 level).

\section{Materials and Methods}

\section{Materials}

Bone and ligament elements were taken from the fresh spine of porcine of the same ages were preserved, and then intervertebral disc-vertebral body functional units from the L4/5 to the L6/S1 level in which soft tissue and muscle had been removed were extracted. These were packaged in a vinyl bag and stored at $-20{ }^{\circ} \mathrm{C}$. Samples were thawed at room temperature immediately before testing. Experiments were conducted at room temperature and physiological saline was occasionally dripped onto specimens to prevent drying.

\section{Threaded interbody fusion cage}

Hollow Modular Anchorage (HMA) cylindrical threaded cages (AESCULAP, Germany) of two sizes $(12 \mathrm{~mm} \times 16 \mathrm{~mm}$ and $12 \mathrm{~mm} \times 20 \mathrm{~mm}$ ) were used. The conical threaded cage was a Novus Lumbar Tapered Interbody Fusion Device (LT-Device: Medtronic-SofamorDanek, USA), and was $13 \mathrm{~mm} \times 11 \mathrm{~mm} \times 12 \mathrm{~mm}$ (Fig. 1).

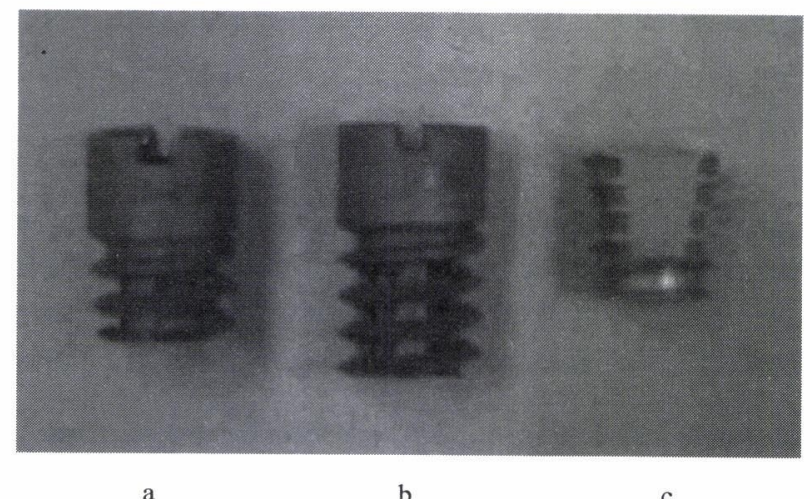

Fig. 1. Interbody fusion cages. From the left-a : HMA $(12 \times 16 \mathrm{~mm}), \mathrm{b}:$ HMA $(12 \times 20 \mathrm{~mm})$, and $\mathrm{c}:$ LT- Device $(13 \times 11 \times 12 \mathrm{~mm})$ 


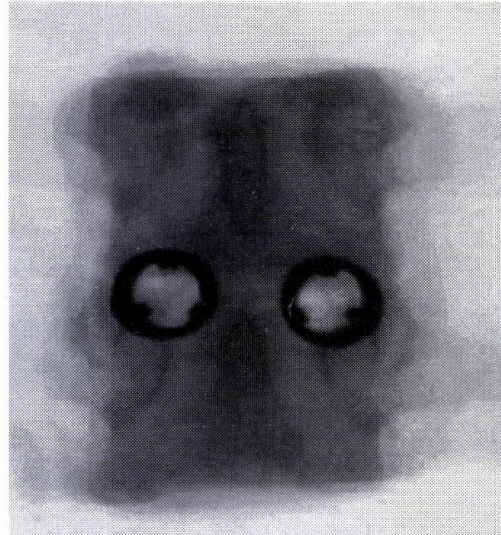

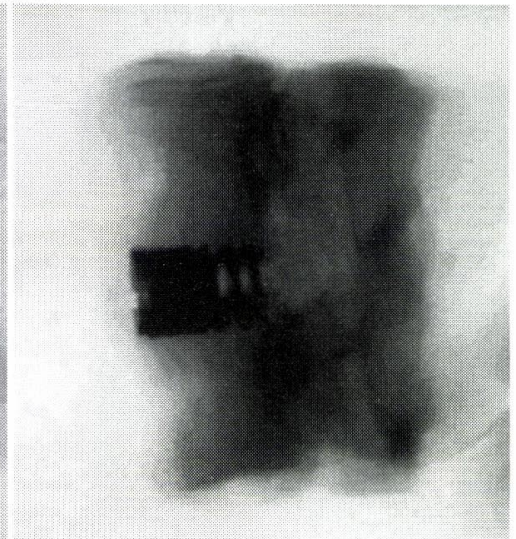

b

Fig. 2. Simple X-ray anteroposterior view (a) and lateral view (b) of the A group (two HMA cages placed anteriorly)

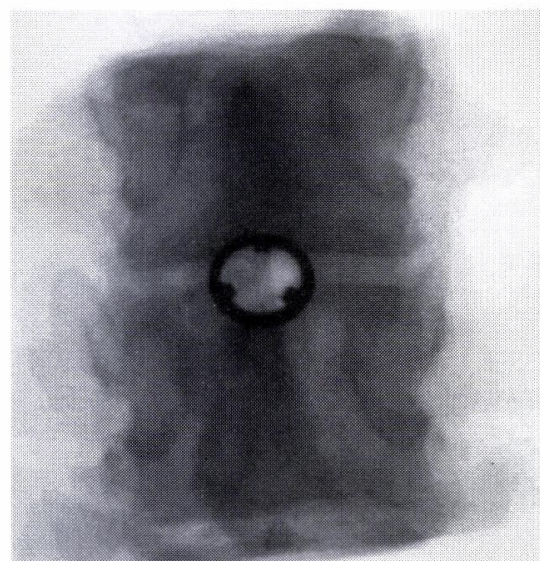

a

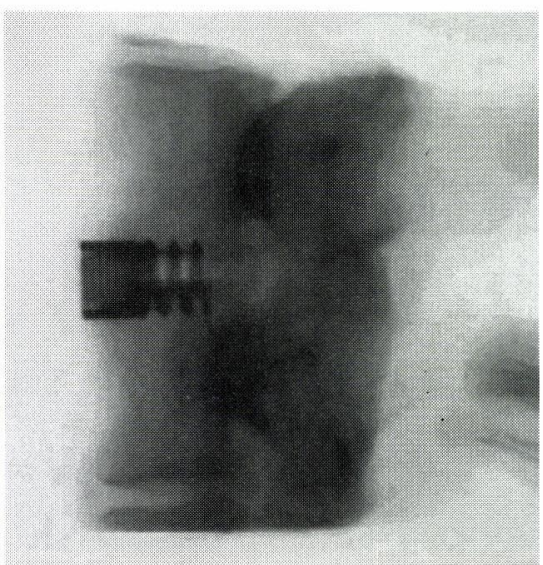

b

Fig. 3. Simple X-ray anteroposterior view (a) and lateral view (b) of the B group (single HMA cage placed centrally and anteriorly)

\section{Study 1}

Fresh porcine L4/5,5/6 spinal functional units were used in the first study, and cages were inserted at the L4/5,5/6 level. In group A two HMA cages were $(12 \mathrm{~mm} \times 16 \mathrm{~mm})$ were placed anteriorly, in group B a single cage $(12 \mathrm{~mm} \times 16 \mathrm{~mm})$ was placed centrally and anteriorly, in group C a single cage $(12 \mathrm{~mm} \times 20 \mathrm{~mm})$ was placed laterally, and in group D a single cage $(12 \mathrm{~mm} \times 20 \mathrm{~mm})$ was placed anterolaterally. Simple X-rays of each insertion method are shown in Figs. 2 to 5. 


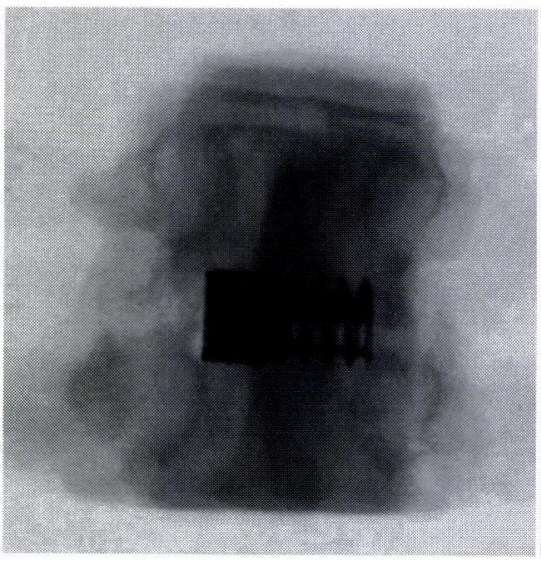

a

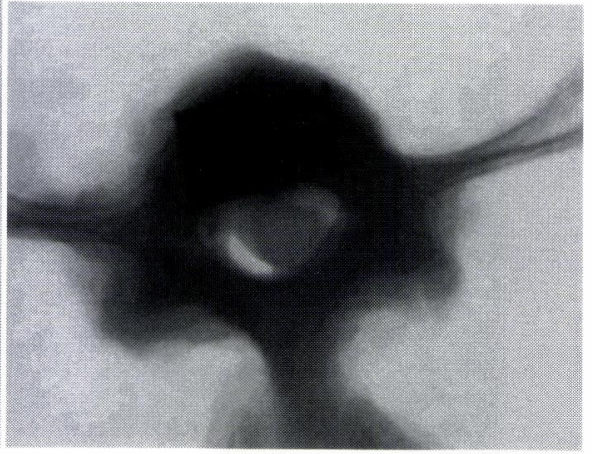

b

Fig. 4. Simple X-ray anteroposterior view (a) and axial view (b) of the $\mathrm{C}$ group (single HMA cage placed laterally)

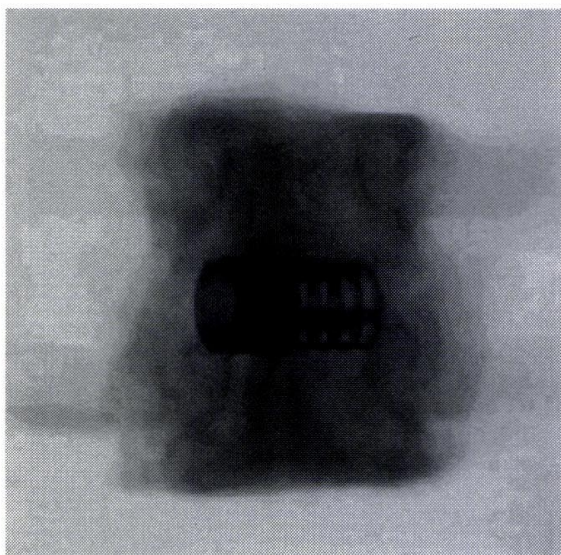

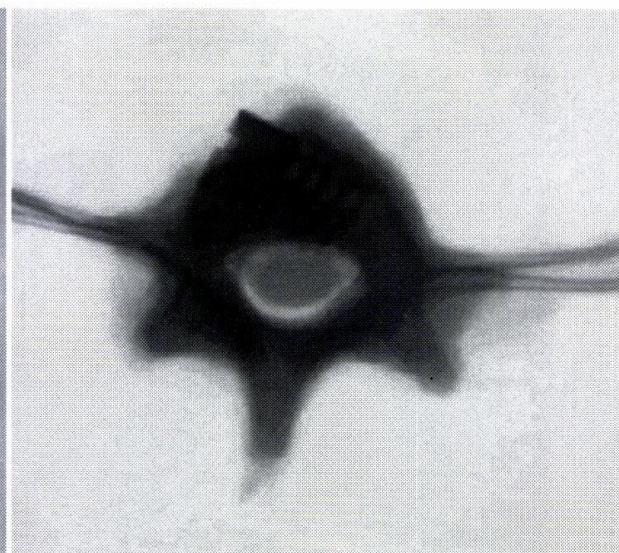

b

Fig. 5. Simple X-ray anteroposterior view (a) and axial view (b) of the D group (single HMA cage placed anterolaterally)

\section{Study 2}

Fresh porcine L6/S1 spinal functional units were used in the second study, and cages were inserted at the L6/S1 level. In the $\mathrm{H}-1$ group an HMA $(12 \mathrm{~mm} \times 16 \mathrm{~mm})$ was placed centrally and anteriorly, and in the L-1 group an LT-Device $(13 \mathrm{~mm} \times 11 \mathrm{~mm} \times 12 \mathrm{~mm})$ was placed in the same manner. In the $\mathrm{H}-2$ group an HMA $(12 \mathrm{~mm} \times 16 \mathrm{~mm})$ was placed anteriorly and in the L-2 group an LT-Device $(13 \mathrm{~mm} \times 11 \mathrm{~mm} \times 12 \mathrm{~mm})$ was placed anteriorly. Simple X-rays of each insertion condition are shown in Figs. 6 to 9.

The control for both studies was a group with no cages installed. 


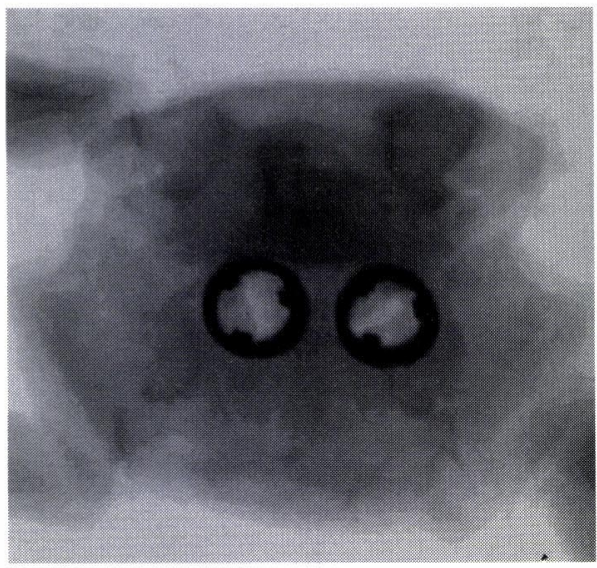

a

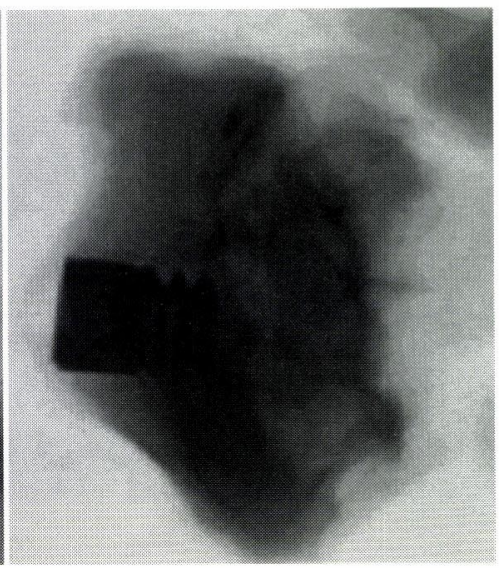

b

Fig. 6. Simple X-ray anteroposterior view (a) and lateral view (b) of the H-2 group (two HMA cages placed anteriorly)

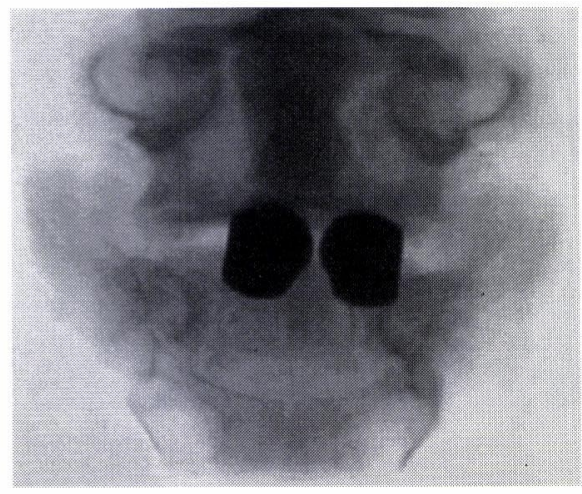

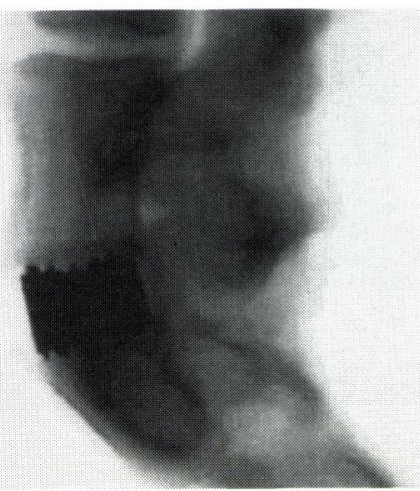

b

Fig. 7. Simple X-ray anteroposterior view (a) and lateral view (b) of the L-2 group (two LT-Device placed anteriorly)

\section{Biomechanical test}

Craniocaudal vertebral bodies were fixed with resin on a metallic pedestal the authors made and an Instron universal testing machine (Autograph: AGS-G100kN, Shimadzu, Kyoto) was installed on the lower pedestal. Four types of load action-flexion, extension, lateral bending, and torsion-were generated using a wire on pulley installed on the upper pedestal (Fig. 10). The diameter of the pulley (Fig. 11) was $50 \mathrm{~mm}$ for flexion, extension, and lateral bending and $80 \mathrm{~mm}$ for torsion at the L4/5,5/6 level and $60 \mathrm{~mm}$ and $86 \mathrm{~mm}$ at the L6/S1 level. The weight of the upper pedestal was about $70 \mathrm{~N}$ and the compression loading about $30 \mathrm{~N}$. The angle of rotation of the pulley was measured via an image sensor (CV-550, Keyence, Osaka), which consisted of a CCD camera and image analyzer. Two given points were first set in the initial screen shot by the CCD camera and this data was 


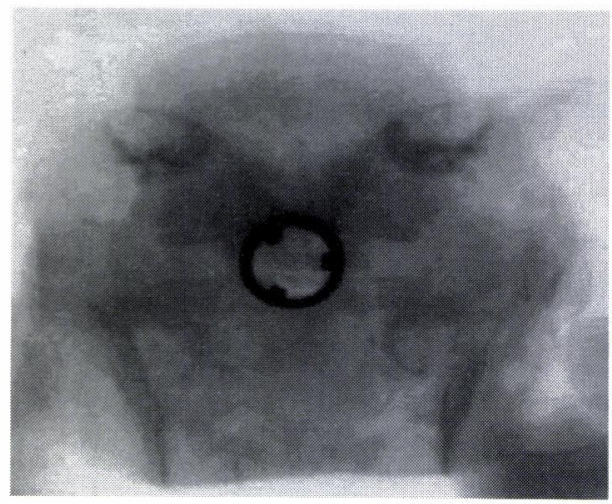

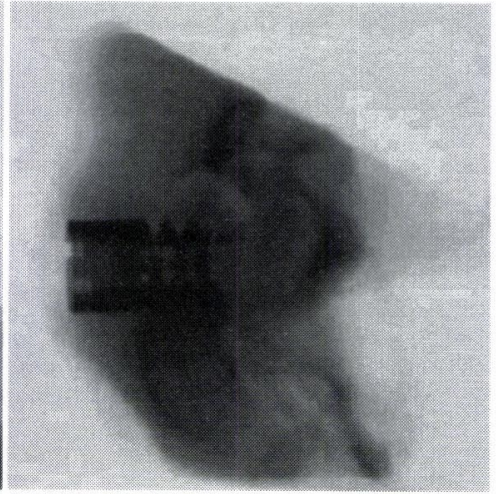

Fig. 8. Simple X-ray anteroposterior view (a) and lateral view (b) of the H-1 group (single HMA cage placed centrally and anteriorly)

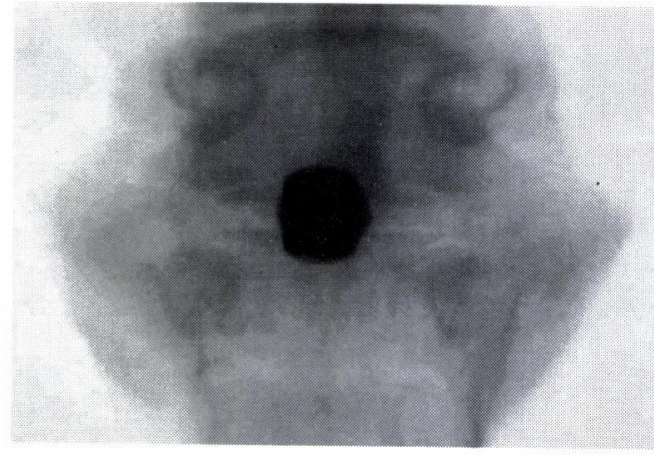

a

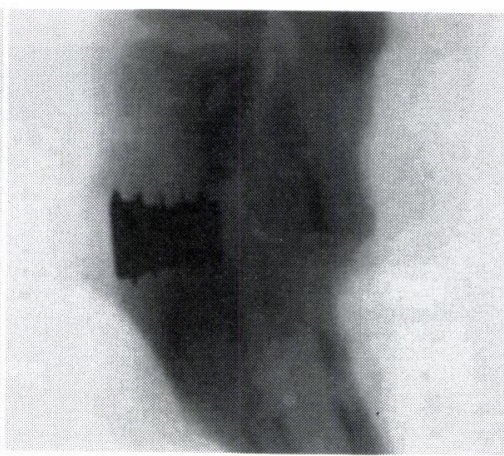

b

Fig. 9. Simple X-ray anteroposterior view (a) and lateral view (b) of the L-1 group (single LT-Device placed centrally and anteriorly)

recorded in the image analyzer. Respective coordinates were measured via tracking by an image analyzer of the movement of two points during filming. In the current testing, the center point of the pulley and, as markers, two places about $20 \mathrm{~mm}$ away were set and the angle of rotation was determined from respective coordinates. The loading moment during flexion, extension, and lateral bending was supplied up to $2.5 \mathrm{~N} \cdot \mathrm{m}$ at the $\mathrm{L} 4 / 5,5 / 6$ level and up to $1.8 \mathrm{~N} \cdot \mathrm{m}$ at the $\mathrm{L} 6 / \mathrm{S} 1$ level; during torsion, it was supplied up to $4 \mathrm{~N} \cdot \mathrm{m}$ and $2.5 \mathrm{~N}$. $\mathrm{m}$. The loading rate was $1 \mathrm{~mm} / \mathrm{min}$; a cycle of loading and unloading was repeated 2-3 times and the stiffness value $(\mathrm{N} \cdot \mathrm{m} / \mathrm{deg})$ was determined from the slope where the moment-angle relationship was stable (Fig. 12).

Analysis of variance (ANOVA) and Fisher's PLSD were used for statistical tests and values below the $5 \%$ significance level were taken to indicate a significant difference. 


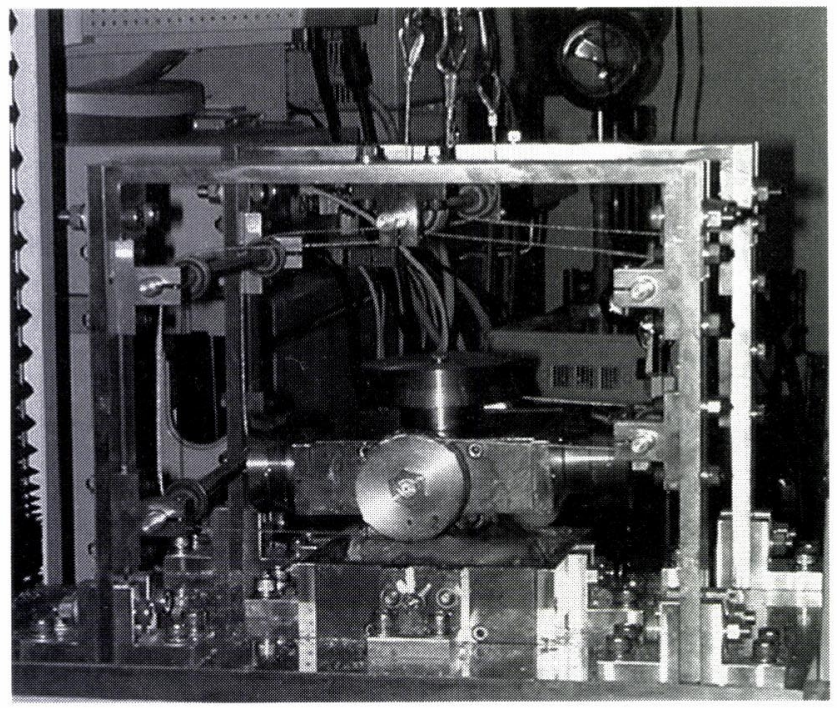

Fig. 10. Test equipment used. Upper and lower vertebral bodies were fixed with resin at the anterior margin of the spinal cord to the metal pedestal at center of a metal box.

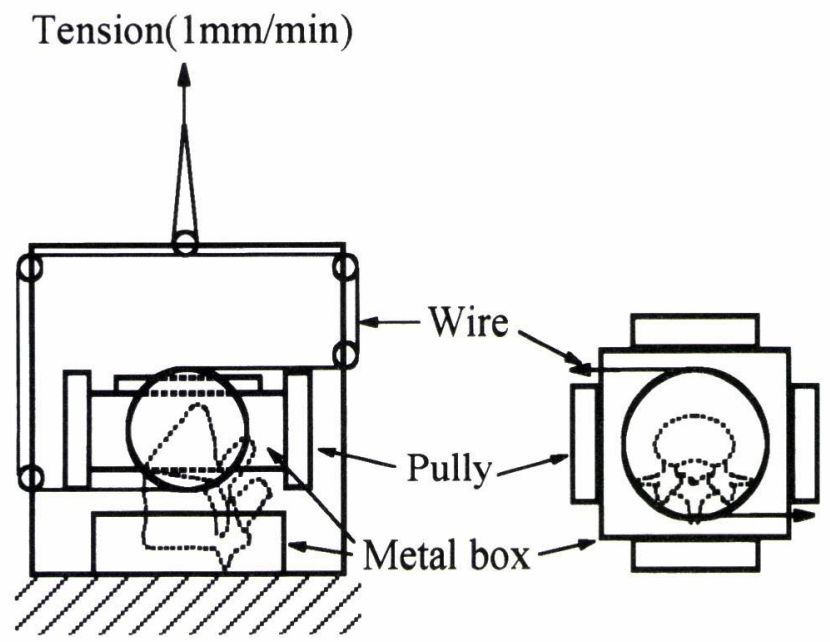

Fig. 11. Load types tested. Four types of load actions-flexion, extension, lateral bending, and torsion-were generated using a wire on a pulley installed on the upper pedestal. 


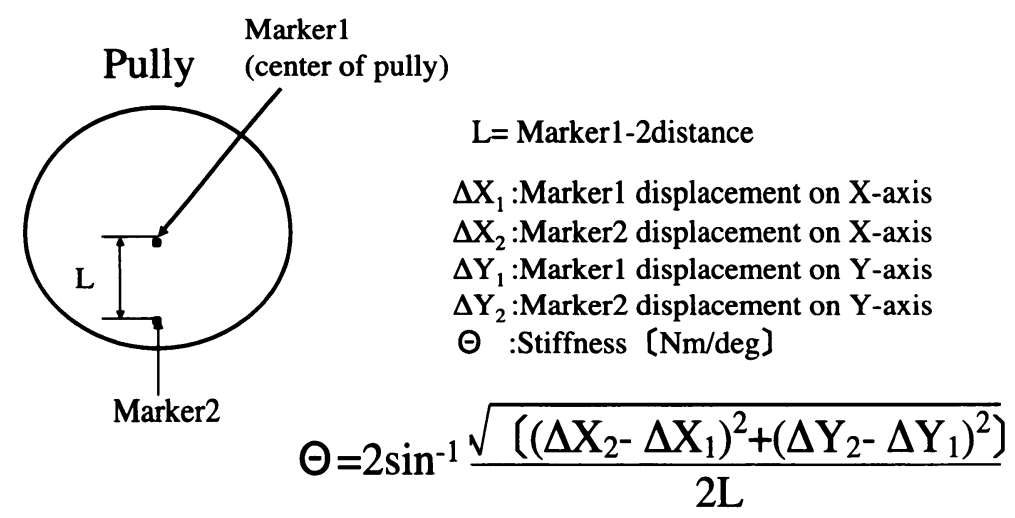

Fig. 12. Calculation of the stiffness value. The center point of the pulley and, as markers, two places about $20 \mathrm{~mm}$ away were set and the angle of rotation was determined from these respective coordinates. A cycle of loading and unloading was repeated 2-3 times and the stiffness value $(\mathrm{N} \cdot \mathrm{m} / \mathrm{deg})$ was calculated from the slope where the moment-angle relationship was stable.

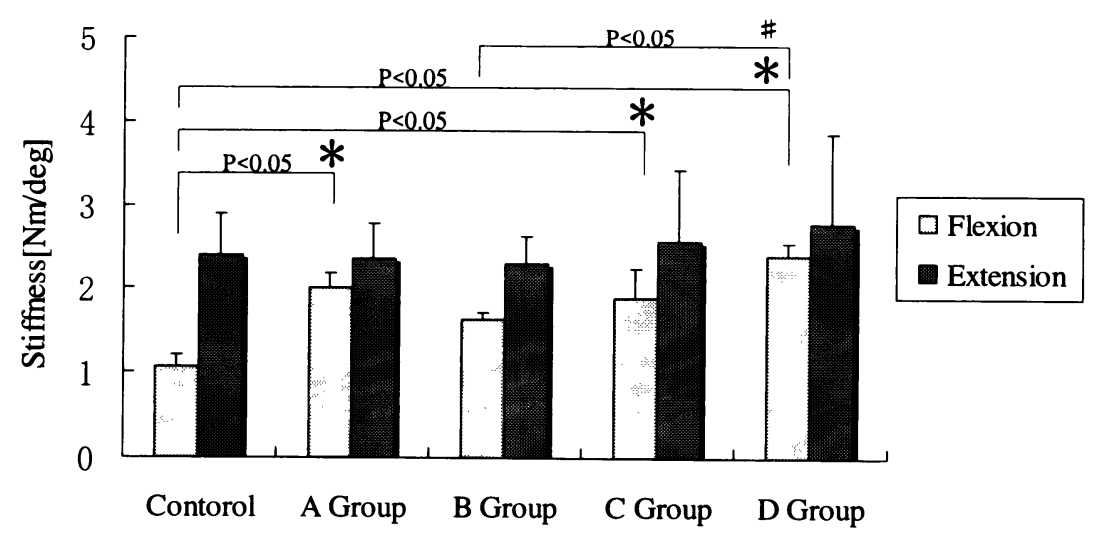

Fig. 13. Comparison of the stiffness value at the L4/5,5/6 level during flexion and extension loading. With flexion, stiffness rose in all of the groups with cages inserted. There was significantly better stiffness in the A, C, and D groups compared to controls $(\mathrm{P}<0.05$ for all $)$. In addition, stiffness was significantly better in the $\mathrm{D}$ group than the B group $(\mathbf{P}<0.05)$. There were no significant differences between any of the groups with extension. Values are mean \pm standard error.

\section{Results}

In individual loading modes, the average stiffness value and standard deviation per cage insertion conditions were compared. The results are shown in Figs. 13 to 16.

\section{Study 1}

Comparison of the average stiffness value for individual loading modes at the L4/5,5/6 level.

With flexion, all of the groups with cages inserted, and this increasee was significantly greater than in the control group in the A, C, and D groups $(\mathbf{P}<0.05$ for all) (Fig. 13). 


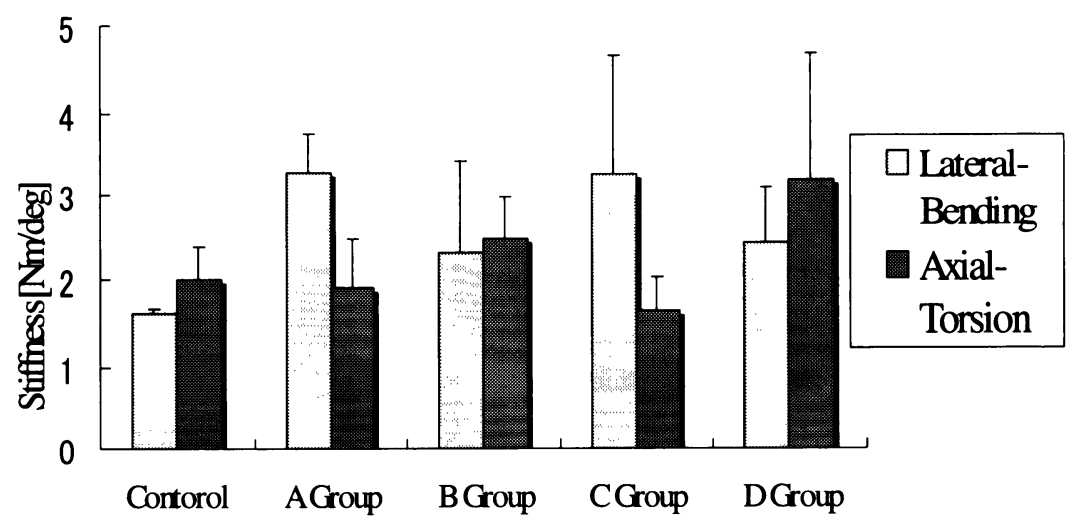

Fig. 14. Comparison of the stiffness value at the L4/5,5/6 level during lateral bending and torsion loading. There were no significant differences between any of the groups.

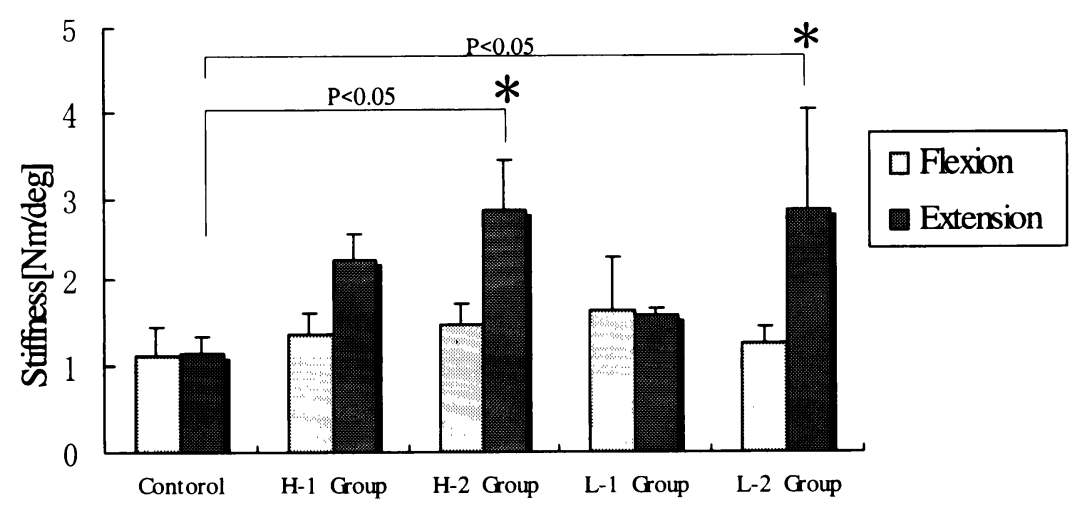

Fig. 15. Comparison of the stiffness value at the L6/S1 level during flexion and extension loading. With flexion, there were no significant differences between any of the groups, but there was a trend for better flexion in the groups that had cages inserted. With extension, there was a trend for better extension in the groups that had cages inserted, and this reached significance in the $\mathrm{H}-2$ and $\mathrm{L}-2(\mathbf{P}<0.05)$.

In addition, stiffness was significantly better in the $\mathbf{D}$ group than the $\mathbf{B}$ group $(\mathbf{P}<0.05)$. There were no significant differences between any of the groups with extension.

There was a tend for improved stiffness in lateral bending in the $\mathbf{A}$ and $\mathbf{C}$ groups, but this did not reach significance (Fig. 14). With torsion, stiffness was greater in the B and $\mathrm{C}$ groups, but again this did not reach significance.

\section{Study 2}

Comparison of the average stiffness value for individual loading modes at the L6/S1 level.

With flexion, there were no significant differences between any of the groups, but there was a trend for better flexion in the groups that had cage inserted (Fig. 15). With extension, there was a trend for better extension in the group that had cages inserted, and this reached significance in the $\mathbf{H}-2$ and $\mathrm{L}-2$ group $(\mathbf{P}<0.05)$.

With lateral bending, stiffness was significantly better in the $\mathrm{H}-2$ group compared to 


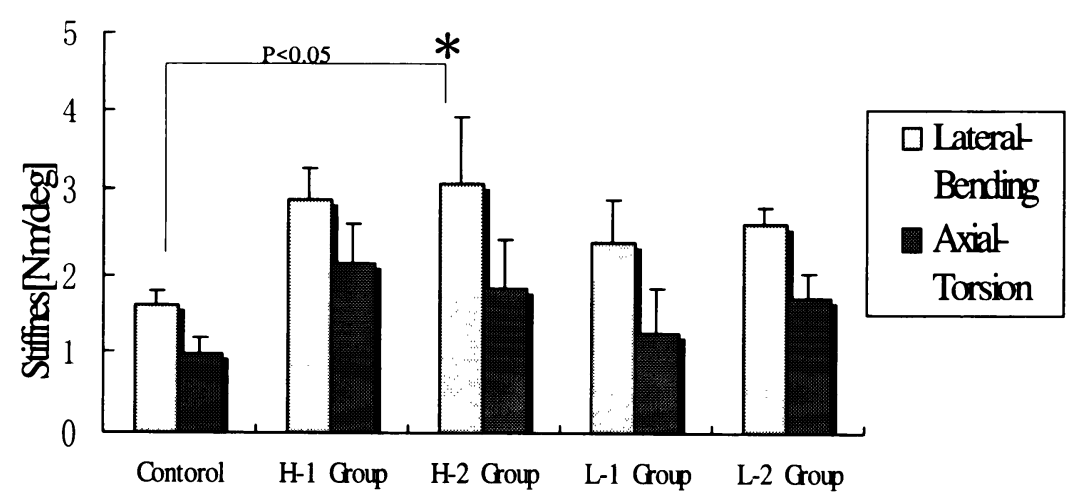

Fig. 16. Comparison of the stiffness value at the L6/S1 level during lateral bending and torsion loading. With lateral bending, stiffness was significantly better in the $\mathbf{H}-2$ group compared to controls $(\mathbf{P}<0.05)$. With torsion, there were no significant differences between any of the groups, but there was a trend for better torsion in the groups that had cages inserted.

controls $(\mathbf{P}<0.05)$ (Fig. 16). With torsion, there were no significant differences between any of the groups, but there was a trend for better torsion in the groups that had cages inserted.

\section{Discussion}

Both anterior and posterior techniques for placement of interbody fusion cages have been shown to give good initial fixation. In the present study, we used an animal model to examine whether the number of cages, the type of cages, or the direction of insertion had any effect on initial fixation.

At the L4/5,5/6 equivalent level in porcine spine we observed an increase in stiffness with flexion and lateral bending, but not with extension and torsion. There was non-significant trend for better stiffness with flexion, extension, and torsion in the single anterolateral insertion group compared to the single anterior insertion group. This may reflect the amount of fibrous ring removed. Ikeda, et $a l^{12)}$ reported that stiffness decreases as the range of fibrous ring removed increases. Zhao, et $a l^{13)}$ created a posterior lumbar interbody fusion (PLIF) model using a threaded cage with the spine of cows and reported that insertion of one cage from the rear has greater stiffness than insertion of two in parallel from the rear. Nydegger, et $a^{14)}$ created an ALIF using a threaded cage with the spines of human cadavers, and stiffness rose significantly in flexion and extension for the group with insertion from the anterolateral compared to the group with no cages inserted. In addition, when comparing the single anterior group and the anterolateral group, a difference in flexion and extension was not noted, although a difference was noted in torsion and lateral bending and stiffness was reported to increase with addition of fixation from the rear. The reason for no improvement in stiffness with extension may be related to anterior longitudinal ligament and fibrous ring damage due to cage insertion ${ }^{4}$. When intervertebral height is obtained and intervertebral discs are extended, local stiffness where fibrous ring and anterior longitudinal ligament tension is high is assumed to increase ${ }^{15,16)}$. In the present study, damage due to cage insertion exceeded this force and therefore there was no 
improvement in stiffness. In addition, the intervertebral joint is substantially involved in extension $^{17)}$ and the effect of a cage inserted is thought to be minimal. The fact that there was no improvement in stiffness with rotation may be a result of the minimal involvement of anterior elements in torsion but greater involvement of the intervertebral joint ${ }^{7,13)}$. Thus, insertion of cage from anterior, should have minimal effects on stiffness. While two cages are often inserted from anterior, sufficient fixation may be expected from the insertion of only one cage from this direction.

At the L6/S1 level, there was significantly better stiffness with extension in the group with the two cylindrical and conical cages inserted. With two cylindrical cages, stiffness rose significantly in lateral bending as well. Tencer, et $a l^{7)}$ created an ALIF model using two cylindrical and conical threaded cages in bovine lumbar vertebrae, and found that the angle of rotation decreased significantly compared to a no-insertion control with the cylindrical cage with lateral bending, and with the conicalcage with extension/lateral bending. In addition, Nibu, et $a l^{3,18)}$, using an ALIF with a BAK cage and a shell-shaped cage in spines of human cadavers, found that and, stiffness rose with flexion, lateral bending, and torsion, but decreased with extension, and the neutral zone and range of motion (ROM) decreased or was equal to that of the group with no cages inserted. We also observed that the shape of the cage had no significant effect on stiffness with flexion, lateral bending, and torsion.

We observed in increase in stiffness with extension for both cages compared to the control. Zhao, et $a l^{13)}$ reported that fixation of the intervertebral joint in an extension position in a PLIF model of the bovine spine has increasing stiffness with respect to extension. When considering physiological lordosis of the lumbar vertebrae and the lumbosacral angle, the upper vertebral body end plate is relatively parallel with intervertebral discs at the human L3/4,4/5 level; in contrast, the L5 lower end plate and S1 upper end plate open slightly in front with intervertebral discs at the human L5/S1 level. That is, as a result of lordosis of lumbar vertebrae increasing because of cage insertion, the fact that it reached a fixed position in a state close to an extension position is because it substantially exceeded the decrease in stiffness due to fibrous ring damage. Our results suggest that initial fixation can be obtained with either a cylindrical or conical cage with intervertebral discs at the human L5/S1 level ${ }^{7)}$, although when considering the intervertebral shape, posterior over-reaming of the upper and lower end plates is a concern when a cylindrical cage is used, so a conical cage that matches the intervertebral shape may be more appropriate. Further study is needed to determine why there was no improvement in stiffness with flexion.

These results suggest that sufficient initial fixation can be obtained even with one cylindrical cage inserted from the anterior for interbody fusion with intervertebral discs at a relatively parallel level ${ }^{13)}$. However, a conical cage matching the intervertebral shape may give better at the human L5/S1 level, where intervertebral discs are open at the front.

The spine currently used is porcine and differs somewhat from that of humans, although these research results may be beneficial for future technique selection for interbody fusion in humans.

\section{References}

1) Dennis S, Watkins R, Landaker S, William D and Springer D : Comparison of disc space heights after anterior 
lumbar interbody fusion. Spine $14: 876-878$ (1989)

2) Ray CD : Threaded titanium cage for lumbar interbody fusion. Spine 22 : 667-680 (1997)

3) Nibu K, Panjabi MM, Oxland T and Cholewicki J : Multidirectional stabilizing potential of BAK interbody spinal fusion system for anterior surgery. J Spinal Disord $10: 357-362$ (1997)

4) Oxland TR, Hoffer Z, Nydegger T, Rathonyi GC and Nolte LP: A comparative biomechanical investigation of anterior lumbar interbody cage : Central and bilateral approaches. J Bone Joint Surg 82A : 383-393 (2000)

5) Sandhu HS, Turner S, Kabo M, Kanim LEA, Liu D, Delamarter RB and Dawson EG : Distractive properties of threaded interbody fusion device; An in vivo model. Spine 21 : 667-680 (1996)

6) Lund T, Oxland TR, Jost B, Cripton P, Grassmann S, Etter C and Notle LP: Interbody cage stabilization in lumbar spine: Biomechanical evaluation of cage design, posterior instrumentation and bone density. $J$ Bone Joint Surg 80(B) : 351-359 (1998)

7) Tencer AF, Hampton D and Eddy S: Biomechanical properties of threaded inserts for lumbar interbody spinal fusion. Spine 20 : 2408-2414 (1995)

8) Brodke DS, Dick JC, Kunz DN, McCabe R and Zdeblick TA : Posterior lumbar interbody fusion. Spine 22 : 26-31 (1997)

9) Oxland TR and Lund T: Biomechanics of stand-alone cages and cages in combination with posterior fixation : a literature review. Eur Spine J 9(Suppl 1): S95-S101 (2000)

10) Glazer PA, Colliou O, Lotz JC and Bradford DS : Biomechanical analysis of lumbosacral fixation. Spine 21 : 1211-1222 (1996)

11) Kanayama M, Cunningham BW, Haggerty CJ, Abumi K, Kaneda K and McAfee C: In vitro biomechanical investigation of the stability and stress-shielding effect of lumbar interbody fusion devices. $J$ Neurosurg (2 Suppl) 93 : 259-265 (2000)

12) Ikeda $M$, Abe $S$, Washio $T$, Hra $T$ and Hasegawa $K$ : An experimental study for lumbar interbody fusion with threaded fusion cage. J Jpn Soc Clin Biomech $19: 67-72$ (1998) (in Japanese)

13) Zhao J, Hai Y, Ordway NR, Park CK and Yuan HA : Posterior lumbar interbody fusion using posterolateral placement of a single cylindrical threaded cage. Spine $25: 425-430$ (2000)

14) Nydegger T, Oxland TR, Hoffer $Z$, Cottle $W$ and Nolte LP: Does anterolateral cage insertion enhance immediate stabilization of the functional spinal unit? A biomechanical investigation. Spine 26 : 2491-2497 (2001)

15) Goodwin RR, James KS, Daniels AU and Dunn HK: Distraction and compression loads enhance spine torsional stiffness. J Biomech 27 : 1049-1057 (1994)

16) Rapoff AJ, Ghanayem AJ and Zdeblick TA : Biomechanical comparison of posterior lumbar interbody fusion cage. Spine 22 : 2375-2379 (1997)

17) Lorenz M, Patwardhan A and Vanderby $\mathbf{R} \mathrm{Jr}$ : Load-bearing characteristics of lumbar facets in normal and surgically altered spinal segments. Spine $8: 122-130$ (1983)

18) Nibu K, Panjabi MM, Oxland T and Cholewicki J : Intervertebral disc distraction with a laparoscopic anterior spinal fusion system. Eur Spine $J$ : 142-147 (1998)

[Received December 12, 2003 : Accepted January 7, 2004] 